\title{
The Influence of Gluteus Maximus on Transverse Plane Tibial Rotation
}

\section{Abstract}

There is a common clinical belief that transverse plane tibial rotation is controlled by the rearfoot. Although distal structures may influence the motion of the tibia, transverse plane tibial rotation could be determined by the proximal hip musculature. Cadaver studies have identified gluteus maximus as having the largest capacity for external rotation of the hip. This study was therefore undertaken to investigate the effect of gluteus maximus on tibial motion. Kinematic data were collected from the foot and tibia along with EMG data from gluteus maximus for 17 male subjects during normal walking. A number of kinematic parameters were derived to characterise early stance phase. Gluteus maximus function was characterised using RMS EMG and EMG on/off times. No differences in muscle timing were found to be associated with any of the kinematic parameters. In addition, no differences in gluteal activation levels were found between groups of subjects who had different amounts of tibial rotation. However, there was a significant difference $(P<0.001)$ in

gluteus maximus activation when groups were defined by the time taken to decelerate the tibia (time to peak internal velocity). Specifically, subjects with greater gluteus maximus activity had a lower time to decelerate the tibia. We suggest that a high level of gluteus maximus activity results in a larger external torque being applied to the femur, which ultimately leads to a more rapid deceleration of the tibia.

Key Words: Tibial rotation, Rearfoot pronation, Gluteus maximus, EMG 


\section{The Influence of Gluteus Maximus on Transverse Plane Tibial Rotation}

\section{Introduction}

A coupling mechanism exists within the ankle joint complex which enables the transfer of pronation/supination to axial tibial rotation [1, 2]. This coupling mechanism arises from the articulations within the ankle, subtalar and midtarsal joints [3]. As the subtalar joint is inclined approximately $45^{\circ}$ from the horizontal, calcaneal eversion transfers into a similar amount of internal tibial rotation [1, 4]. It is commonly believed that, through this mechanism, the foot motion controls the transverse plane rotation of the tibia and subsequently the entire lower limb. Abnormal magnitude or timing of foot pronation is believed to result in abnormal internal tibial rotation and to be associated with a number of musculoskeletal pathologies, including patello-femoral pain [5, 6], plantar fascitis [7] and Achilles tendonitis [8].

Transverse plane tibial motion is determined by torques applied both proximally and distally. These torques are generated by muscle-tendon forces, ligamentous constraints and external forces, such as the ground reaction force. If the torques acting at the proximal end of the tibia are larger than those acting at the distal end, the tibia is considered to be driven proximally. Conversely, larger distal forces would mean more distal control. By using a 'power-flow' analysis, Bellchamber and van den Bogert [9] found evidence for proximal control during walking in late stance phase. This result demonstrates that tibial motion is not always controlled by the structures surrounding the ankle. As the muscles surrounding the knee joint have little capacity for transverse plane tibial rotation, proximal control may originate from the hip musculature. Although there have been some studies attempting to relate foot 
structure to tibial kinematics [10], there is a lack of published studies investigating the effect of hip biomechanics and specifically the potential role of the hip musculature. Therefore, further research is required to establish whether specific hip muscles could have a significant influence on transverse plane tibial rotation. Any research which could demonstrate a link between hip muscle function and tibial rotation has the potential to influence clinical practice. Although, the traditional view is that abnormal rotation should be corrected at the ankle, it may be possible to change transverse plane tibial rotation by focusing on muscle activity at the hip.

At heelstrike the ground reaction force, acting on the plantar aspect of the foot, causes the rearfoot to pronate and the tibia to internally rotate $[11,12]$. This motion continues to the point of peak internal rotation, which occurs between $20-25 \%$ stance during normal walking [11] (figure 1a). During this period it is possible that specific hip muscles could act eccentrically to apply an external rotation moment to the femur and thus decelerate the internal rotation of the tibia. During the initial $20-25 \%$ of stance, the hip is flexed approximately $25^{\circ}$ [13]. In this position, activation across all compartments of either gluteus medius or minimus would produce limited transverse plane femoral rotation [14]. The same is true for the combined effect of the hamstrings or the adductors [15]. The situation is different for gluteus maximus, which, due to is anatomical position, has a large capacity for external rotation [14]. Given the relative strength of this muscle, the fact it is active during early stance [16] and its large capacity for external rotation [14], it has significant potential to affect tibial kinematics. 
Soft tissue motion artefact is known to significantly influence derived kinematics for transverse plane lower extremity motion [17]. In particular a number of studies have shown that errors from skin-mounted marker based estimates of thigh rotation are similar in magnitude to the true motions [18-20]. As such it has been concluded that skin-mounted marker systems are not appropriate for representing the transverse plane motion of the femur or the knee joint $[17,20]$. Soft tissue artefact has been found to be considerably less for the tibia [18, 19]. Moreover, a recent study demonstrated that soft tissue artefact can be reduced by using a constrained marker cluster, placed distally on the shank [21]. Using this suggested marker protocol, it may be possible identify differences in tibial kinematics between subjects and to relate this to hip muscle function.

We undertook this study as a first step towards understanding the effect of the proximal hip musculature on the transverse plane rotation of the tibia. Specifically, we aimed to address the following research questions: (a) is transverse plane tibial rotation influenced by the degree of activation of gluteus maximus (b) is transverse plane tibial rotation influenced by the time of onset/duration of activity of gluteus maximus.

\section{Methods}

\subsection{Data Collection}

The study was restricted to male subjects as pilot work on 10 female subjects showed that EMG signals, collected from gluteus maximus, were as low as $10 \%$ of those in males. It is likely such low signals are the result of the thicker layer of subcutaneous fat in females subjects. Data were collected on 34 limbs from 17 
subjects (mean age: $24 \pm 5$ years, mean mass: $80 \pm 12 \mathrm{Kg}$ ) who had no musculoskeletal complaints. Each subject provided written consent to participate and ethical approval had been granted by the institutional committee.

Data were collected during 10 barefoot walking trials $\left(1.25+/-0.1 \mathrm{~ms}^{-1}\right)$, with walking speed measured using photocells placed 5m apart. Barefoot walking was used, instead a shod condition, as footwear has the potential to influence rearfoot motion and therefore transverse plane tibial motion. Ground reaction force data were collected (Kistler, $1000 \mathrm{~Hz}$ ) to enable accurate identification of stance phase. Tibial kinematic data were collected using ten Qualisys motion capture cameras $(100 \mathrm{~Hz})$. To minimise the effects of skin movement artefact we adopted the protocol for tibial marker placement recommended by Manal et al. (2000). A 9cm square Velcro ${ }^{\mathrm{TM}}$ backed rigid plate, with four $9 \mathrm{~mm}$ markers, was attached to the lateral-distal aspect of the shank and was held in place using an underwrapped elasticised bandage. To define the tibial local co-ordinate frame and a standard reference orientation $\left(0^{\circ}\right)$, a static standing trial was taken in which additional markers were attached to the malleoli and the epicondyles of the femur.

EMG data were collected using a Noraxon Telemyo system $(3000 \mathrm{~Hz})$ with electrodes placed bilaterally on gluteus maximus according to the guidelines in the European SENIAM standard [22]. After data collection the gait EMG data was band pass filtered between $20-500 \mathrm{~Hz}$. Although it is customary to use a maximal voluntary contraction to normalise EMG data, it has been shown by Yang and Winter [23] that submaximal contractions are more reliable. In particular, a sub-maximal contraction has been advocated for gluteus maximus due to the difficulty in isolating this muscle 
from the other hip extensors [24]. In this study a reference sub-maximal isometric contraction task was used in which the subject lay prone, with the knee flexed at $90^{\circ}$ and the hip maintained in neutral flexion-extension. In this position the hamstrings are assumed to be in a position of insufficiency and unable to contribute to the extensor hip moment. During data collection each subject was instructed to relax as much as possible whilst maintaining the hip in neutral flexion-extension with the contralateral limb and the upper body supported on the testing plinth. After several practices, four trials of this task were recorded (minimum 3 seconds of data).

\subsection{Data Processing}

The 3D coordinate data from the static trial was used to calculate joint centres for the ankle and knee. The ankle and knee centres were used to define a longitudinal tibial axis $(Z)$. A medial lateral axis $(Y)$ was then defined, which was perpendicular to the Z-axis and in the plane formed by the Z-axis and the line joining the malleoli markers. The anterior/posterior axis $(X)$ was perpendicular to both other axes. The motion data were filtered at $6 \mathrm{~Hz}$ Butterworth, to maintain consistency with Manal et al. [21], and the angle of the tibia with respect to the laboratory calculated using Cardan angles in $\mathrm{XYZ}$ sequence. From this data, tibial velocity and acceleration were obtained as the first and second derivative respectively. Stance was defined when the vertical force data was above the mean plus 2 SD of the baseline noise, typically $3-5 \mathrm{~N}$.

To quantify transverse plane tibial motion, it was necessary to derive a number of parameters. Range of motion (ROM), over the first $50 \%$ of stance phase, and time to peak internal rotation (tPIR [\% stance]) were derived as they relate to commonly 
reported clinical concepts of foot and leg function [25]. In addition, peak internal velocity (PIV) and time to peak internal velocity (tPIV [\% stance]) were derived. These four parameters are illustrated in figures 1a-c which show typical curves for tibial angle, velocity and acceleration $A$ value for each of the four kinematic parameters was derived from each trial and the final value taken as the mean over the ten trials. In this way four parameters were derived for each limb.

The typical pattern of tibial acceleration (figure 1c) shows a rapid transition from internal to external during early stance. The exact point in time at which the tibia experiences zero acceleration is given by the parameter tPIV. Given the direct proportionality between acceleration and net torque, this parameter gives an indication of the magnitude and timing of the external torque applied to the tibia. A larger, more rapidly applied external torque would result in an earlier value for tPIV, whereas a smaller, more slowly applied torque, would result in a later value for tPIV.

To answer our primary research question, which related to the degree of activation of gluteus maximus, muscle activity was quantified using RMS EMG over a fixed time window. There is a electromechanical delay between EMG activity and the development of muscle force [26]. Therefore, it was necessary to adjust the window backwards in time by $50 \mathrm{~ms}$. The time window was taken as $0-20 \%$ stance as this was the period within which the 4 kinematic events typically occurred. Investigation showed that if a shorter window was used, for example $0-10 \%$, there was too much between trial variability in the RMS value. This was most likely due to the limited number of EMG samples which are contained within such a short time window. As with the kinematic parameters, the RMS EMG was calculated for each trial and then 
averaged over all 10 trials. This value was then expressed as a \% of the mean RMS reference EMG signal from the sub maximal contraction task. This final RMS parameter was derived for each limb.

To answer our second research question, which related to onset and duration of gluteus maximus activity, we used an approximated generalised likelihood (AGLR) algorithm [27]. This has been argued to be more reliable for determining muscle on/off during gait than threshold criteria [27]. Values for the onset and duration of muscle activity were derived from each of the 10 separate trials and then averaged. Again, this was repeated for each limb. Figure 2 illustrates how the three EMG parameters were derived for a typical EMG signal.

For each research question there were two approaches to statistical analysis of the data. Firstly, correlations were investigated between each of the kinematic parameters and (a) RMS EMG and (b) temporal EMG parameters. Soft tissue motion artefact will result in an associated error in any derived kinematic parameter. Therefore the second test was based on a comparison of two extreme groups, defined according to the nature of each kinematic parameter. Group 1 comprised limbs which had a ROM, PIV, tPIR or tPIV which was smaller/earlier than the mean for the sample - 0.25SD. Group 2 comprised limbs which had ROM, PIV, tPIR or tPIV which was larger/later than the mean for the sample $+0.25 S D$. Independent ttests, with an alpha level of 0.01 , were used to compare (a) normalised RMS EMG and (b) temporal EMG parameters between the two groups. The factor 0.25 was chosen as it meant only 5-6 data points were typically excluded, leaving 12 to 18 
data points in each group. This was enough for statistical comparison and allowed groups to be defined with clear differences in kinematic parameters.

\section{Results}

A weak, but significant correlation, $r=-0.41(P=0.02)$, was found between the degree of gluteus maximus activity, over $0-20 \%$ stance, and tPIV. This showed larger gluteus maximus activity to be associated with a shorter time to reach PIV. The other three kinematic parameters were not found to correlate with the degree of muscle activation (table 1$)$. The group analysis showed a significant difference $(p<0.001)$ in muscle activation only when the groups were defined on tPIV. These groups are identified by the horizontal lines in figure 3. This plot shows larger gluteus maximus activity to be associated with faster tibial deceleration. No differences in muscle activation were found when groups were based on the other three kinematic parameters (table 2).

No significant correlations were found between time of gluteus maximus onset or duration of activity and any of the four kinematic parameters (table 1). Similarly there were no differences in temporal EMG parameters between groups defined on kinematic parameters (table 2). A summary of the means and SD, across all limbs, for each of the kinematic parameters is given in table 3a. Means and SD for each of the three EMG parameters are given in table 3b.

\section{Discussion}

The initial aim of this study was to understand whether transverse plane tibial rotation is influenced by the degree of activation of gluteus maximus. To address this 
question, four kinematic parameters were derived to characterise tibial rotation. Only the time to peak internal rotation velocity (tPIV) was found to correlate with the degree of muscle activation, but the correlation was weak. A further two-group comparison showed higher gluteus maximus activity to be associated with an earlier deceleration of the tibia (earlier tPIV). Extreme values of the other three parameters were not found to be associated with differences in gluteus maximus activation.

The parameter tPIV will decrease as the magnitude of the external torque applied to the tibia increases. Therefore, it is possible that, in subjects who display larger levels of gluteus maximus activity, there is a larger external torque applied to the femur and thus the tibia. This may explain the lower values for tPIV. In subjects who display lower levels of gluteus maximus activity, there may be a smaller torque applied to the tibia which results in a slower deceleration of the tibia.

No difference in gluteus maximus activation was found between groups based on tibial ROM over the first $50 \%$ of stance. This would suggest that, in an asymptomatic population, amplitude of motion during early stance is not regulated by gluteus maximus activity. Instead, it may be determined by other factors such as the anatomical structure of the rearfoot or the constraints at the knee joint. Although PIV will be influenced by the torques applied to the tibia, it will also depend on ROM. Although no difference was found in gluteus maximus activation between groups based on PIV, a higher ROM over early stance was found to correlate with a higher PIV $(r=-0.71, p<0.001)$. This could indicate that tibial velocity is primarily determined by the amplitude of the motion, which may be determined by anatomical factors rather than by gluteus maximus activation levels. 
This study found no link between transverse plane tibial motion and either the time of onset or duration of activity of gluteus maximus. This would suggest that the temporal characteristics of gluteus maximus activation do not significantly influence transverse plane tibial kinematics.

There are two principal limitations to the present study. The first is the use of a skin mounted marker set to measure and characterise the motion of the underlying tibia. Although a more accurate representation of tibial motion could be obtained using bone pins, this approach is unfeasible for large numbers of subjects. In collecting our experimental data a kinematic marker protocol was used which has been demonstrated to minimise soft tissue motion artefact [21]. Even with this protocol, there will still be an error in any derived kinematic parameter. Given this error, we compared muscle activation levels between groups, defined from extreme values of kinematic parameters, as well as investigating simple correlations. From the published literature, it is difficult to establish how soft tissue artefact will affect each of the kinematic parameters. However, it is likely that tPIV would be more affected than simple amplitude measures as it is calculated from a derivative of the angular motion curve. Even given the potential for error in this parameter, a significant difference in gluteus maximus activation was found between groups based on tPIV.

The second principal limitation to the study is the use of EMG techniques to quantify muscle activation levels. Given the dynamic nature of the gluteus maximus contraction during walking, a normalised RMS value will give only an approximate 
indication of muscle activation level. Again, given this potential uncertainty, a twogroup comparison may be more appropriate than a correlation analysis.

The current clinical paradigm suggests that tibial motion is controlled by structures distal to the tibia. This study was undertaken as a first step towards understanding the effect of the proximal hip musculature on transverse plane tibial rotation. Specifically, gluteus maximus was identified as the muscle with the largest capacity for transverse plane hip rotation and its activity quantified during walking. Our data showed that subjects with a rapid deceleration of the tibia had high gluteus maximus activation. This was in comparison to subjects with a slow deceleration of the tibia who had relatively lower gluteus maximus activation. It is possible that a high level of gluteus maximus activation results in a larger external torque being applied to the femur which ultimately leads to a more rapid deceleration of the tibia.

\section{Acknowledgements}

This work was financially supported by the EPSRC, UK, grant number GR/S59710/01.

\section{References:}

1. van den Bogert AJ, Smith GD, Nigg BM. In vivo determination of the anatomical axes of the ankle joint complex: an optimization approach. $\mathrm{J}$ Biomech 1994;27:1477-88.

2. Nigg BM, Cole GK, Nachbauer W. Effects of arch height of the foot on angular motion of the lower extremities in running. J Biomech 1993;26:909-16. 
3. Lundberg A, Svensson OK, Bylund C, Selvik G. Kinematics of the ankle/foot complex - part 3: Influence of leg rotation. 1989;9:304-9.

4. McClay I, Manal K. Coupling parameters in runners with normal and excessive pronation. J Appl Biomech 1997;13:109-24.

5. Callaghan MJ, Baltzopoulos V. Gait analysis in patients with anterior knee pain. Clin Biomech 1994;9:79-84.

6. Tiberio D. The effect of excessive subtalar joint pronation on patellofemoral mechanics: A theoretical model. J Orthop Sports Phys Ther 1987;9:160-5.

7. Marshall $P$. The rehabilitation of overuse foot injuries in athletes and dancers. Clin Sports Med 1988;7:175-91.

8. Clement DB, Taunton JE, Smart GW. Achilles tendinitis and peritendinitis: aetiology and treatment. Am J Sports Med 1984;12:178-84.

9. Bellchamber T, van den Bogert AJ. Contributions of proximal and distal moments to axial tibial rotation during walking and running. J Biomech 2000;33:1397-403.

10. Nigg B, Cole G, Nachbauer W. Effects of arch height of the foot on angular motion of the lower extremities in running. J Biomech 1993;26:909-16.

11. Nester CJ, Hutchins S, Bowker P. Shank rotation: A measure of rearfoot motion during normal walking. Foot Ankle Int 2000;21:578-83.

12. Hunt AE, Smith RM, Torode M, Keenan AM. Inter-segment foot motion and ground reaction forces over the stance phase of walking. Clin Biomech $2001 ; 16: 592-600$.

13. Kadaba MP, Ramakrishnan HK, Wootten ME, Gainey J, Gorton G, Cochran GV. Repeatability of kinematic, kinetic, and electromyographic data in normal adult gait. J Orthop Res 1989;7:849-60. 
14. Delp SL, Hess WE, Hungerford DS, Jones LC. Variation of rotation moment arms with hip flexion. J Biomech 1999;32:493-501.

15. Dostal WF, Soderberg GL, Andrews JG. Actions of hip muscles. Phys Ther 1986;66:351-61.

16. Winter DA, Yack HJ. EMG profiles during normal human walking: stride-tostride and inter-subject variability. Electroencephalogr Clin Neurophysiol $1987 ; 67: 402-11$.

17. Leardini A, Chiari L, Della Croce U, Cappozzo A. Human movement analysis using stereophotogrammetry. Part 3. Soft tissue artifact assessment and compensation. Gait Posture 2005;21:212-25.

18. Cappozzo A, Catani F, Leardini A, Benedetti MG, Croce UD. Position and orientation in space of bones during movement: experimental artefacts. Clin Biomech 1996;11:90-100.

19. Reinschmidt C, van den Bogert AJ, Lundberg A, Nigg BM, Murphy N, Stacoff A, Stano A. Tibiofemoral and tibiocalcaneal motion during walking: external versus skeletal markers. Gait Posture 1997;6:89-109.

20. Fuller J, Liu LJ, Murphy MC, Mann RW. A comparison of lower-extremity skeletal kinematics measured using skin- and pin-mounted markers. Hum Mov Sci 1997;16:219-42.

21. Manal K, McClay I, Stanhope S, Richardson J, Galinat B. Comparison of surface mounted markers and attachment methods in estimating tibial rotations during walking: an in vivo study. Gait Posture 2000;11:38-45.

22. Hermens HJ, Freriks B, Disselhorst-Klug C, Rau G. Development of recommendations for SEMG sensors and sensor placement procedures. J Electromyogr Kinesiol 2000;10:361-74. 
23. Yang JF, Winter DA. Electromyography reliability in maximal and submaximal isometric contractions. Arch Phys Med Rehabil 1983;64:417-20.

24. Worrell TW, Karst G, Adamczyk D, Moore R, Stanley C, Steimel B, Steimel S. Influence of joint position on electromyographic and torque generation during maximal voluntary isometric contractions of the hamstrings and gluteus maximus muscles. J Orthop Sports Phys Ther 2001;31:730-40.

25. Powers CM, Chen PY, Reischl SF, Perry J. Comparison of foot pronation and lower extremity rotation in persons with and without patellofemoral pain. Foot Ankle Int 2002;23:634-40.

26. Sherif MH, Gregor RJ, Liu LM, Roy RR, Hager CL. Correlation of myoelectric activity and muscle force during selected cat treadmill locomotion. J Biomech 1983;16:691-701.

27. Roetenberg D, Buurke JH, Veltink PH, Forner Cordero A, Hermens HJ. Surface electromyography analysis for variable gait. Gait Posture 2003;18:109-17. 
Figure 1a: Typical transverse plane tibial rotation during the stance phase of walking ( $0 \%$ represents heel strike and $100 \%$ toe off). ROM refers to tibial range of motion over $0-50 \%$ stance phase and tPIR to the time taken to reach the point of maximal tibial internal rotation. Adapted from [11].

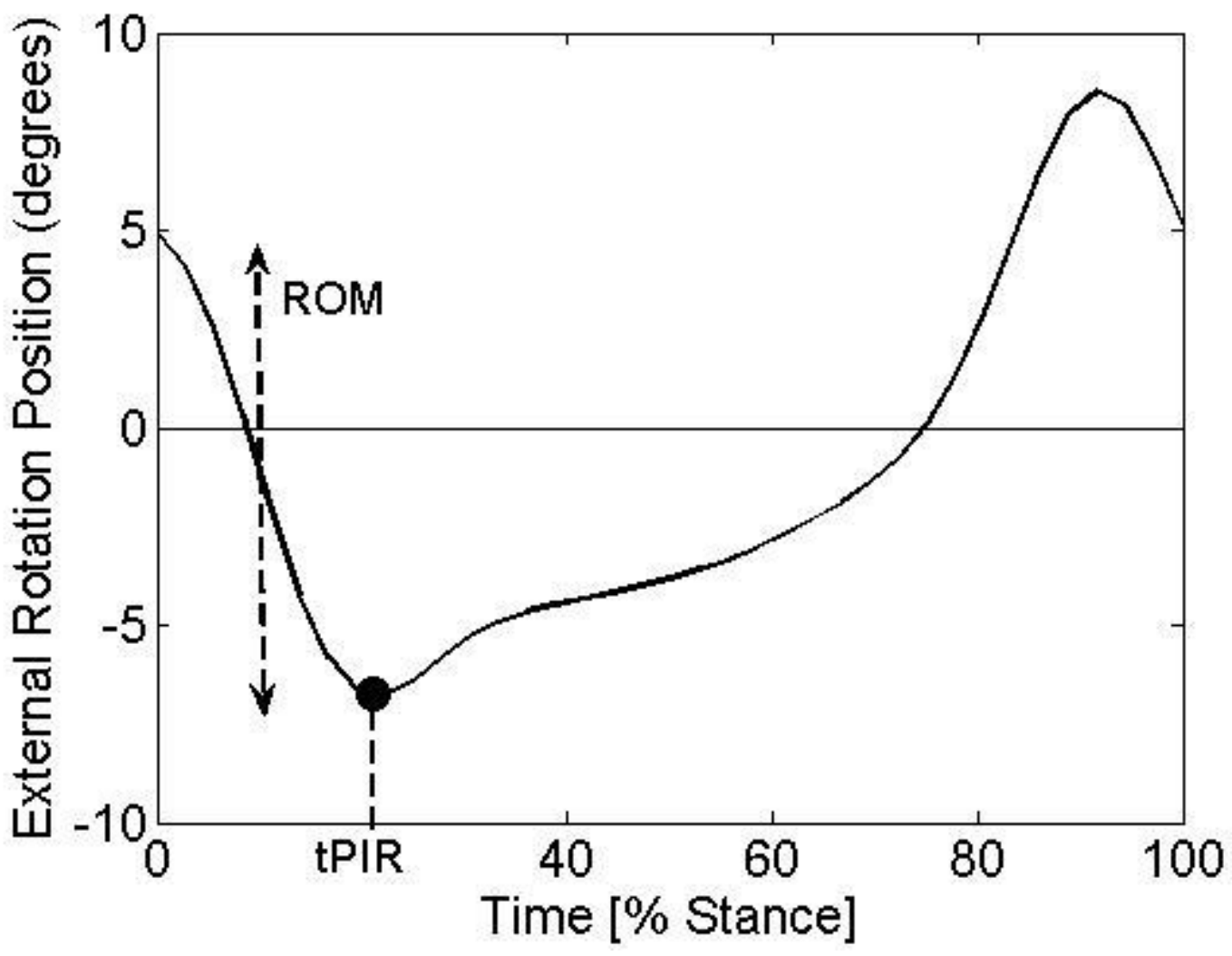


Figure 1b: Typical transverse plane tibial velocity during the stance phase of walking ( $0 \%$ represents heel strike and $100 \%$ toe off). PIV refers to the maximal internal rotation velocity of the tibia and tPIV to the time taken to reach this point. Adapted from [11].

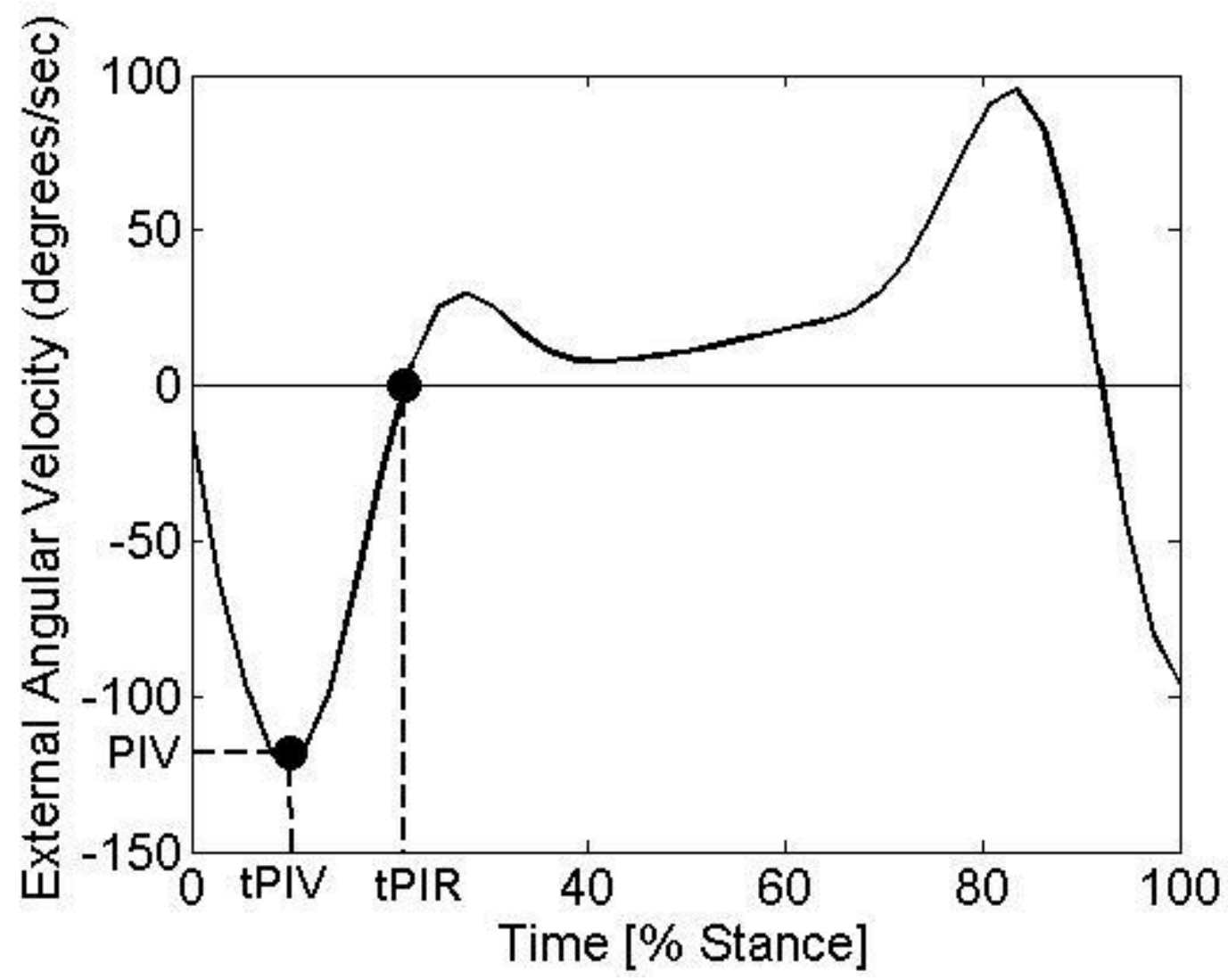


Figure 1c: Typical transverse plane tibial acceleration during the stance phase of walking ( $0 \%$ represents heel strike and $100 \%$ toe off). Adapted from [11].

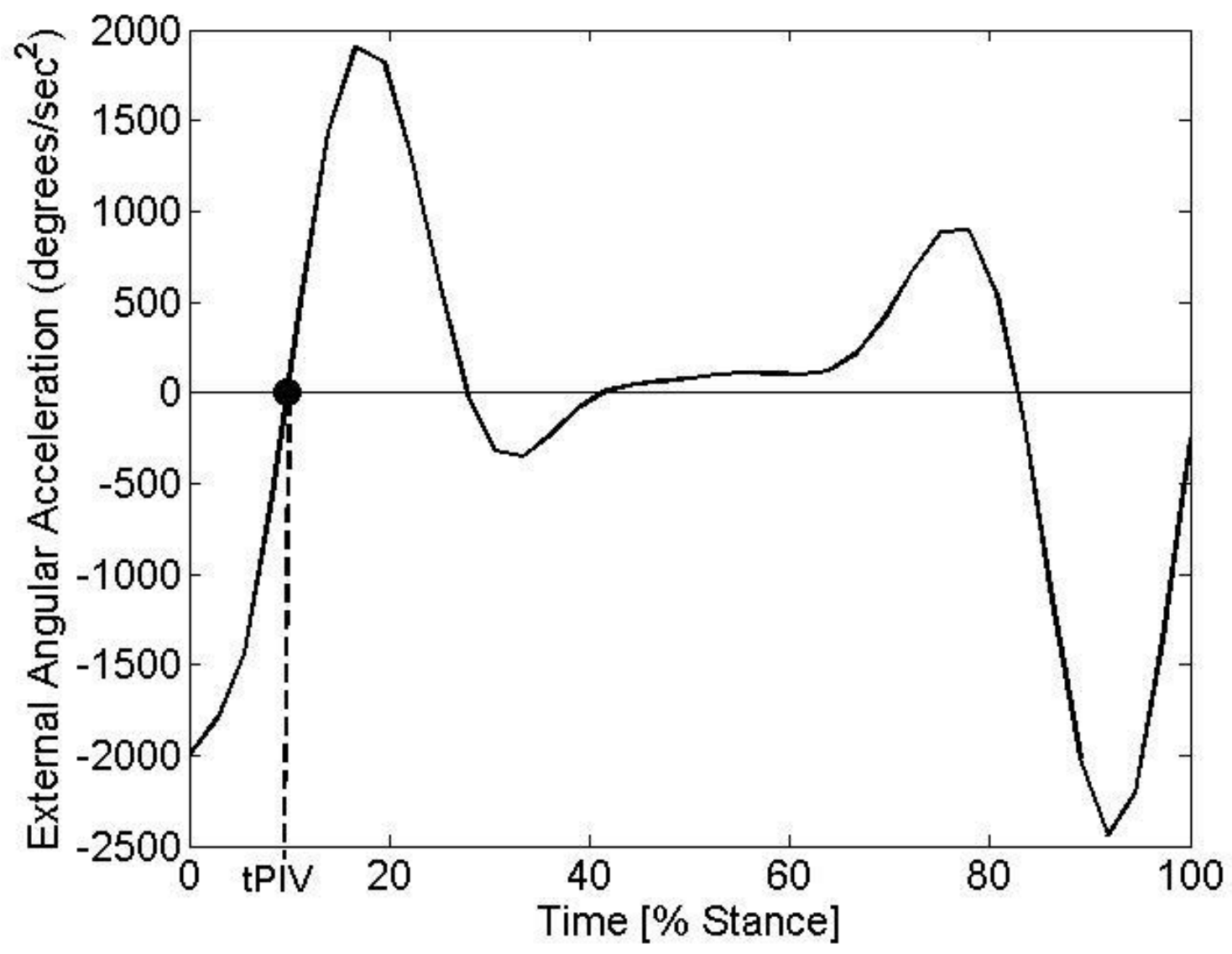


Figure 2: A typical EMG signal represented over the stance phase of walking $(0 \%$ represents heel strike and $100 \%$ toe off). The plot has been annotated to show how the three EMG parameters are derived from each EMG signal. These parameters are a) RMS EMG over 0-20\% stance (adjusted backwards in time to account for electromechanical delay), b) muscle onset time and c) duration of muscle activity.

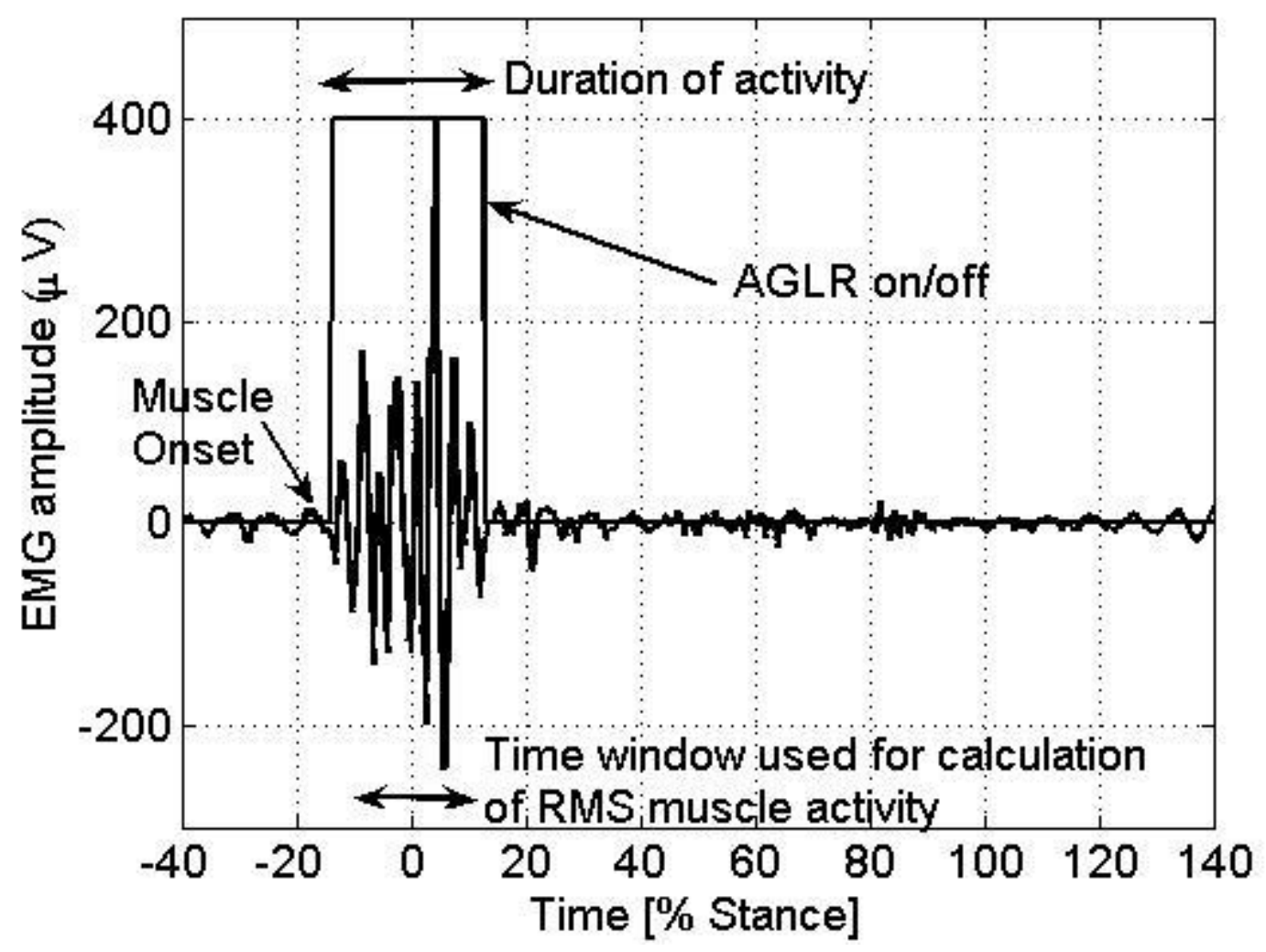


Figure 3: The relationship between the time to peak internal rotation velocity of the tibia and the degree of gluteal activation. Data is for 34 limbs (left and right limbs of 17 subjects) $(r=-0.41, p=0.02)$. The two horizontal lines identify the data 0.25 SD above and below the sample mean of time to peak internal velocity of the tibia. The points above the upper line correspond to the group with late tPIV and the points below the lower line to the group with early tPIV.

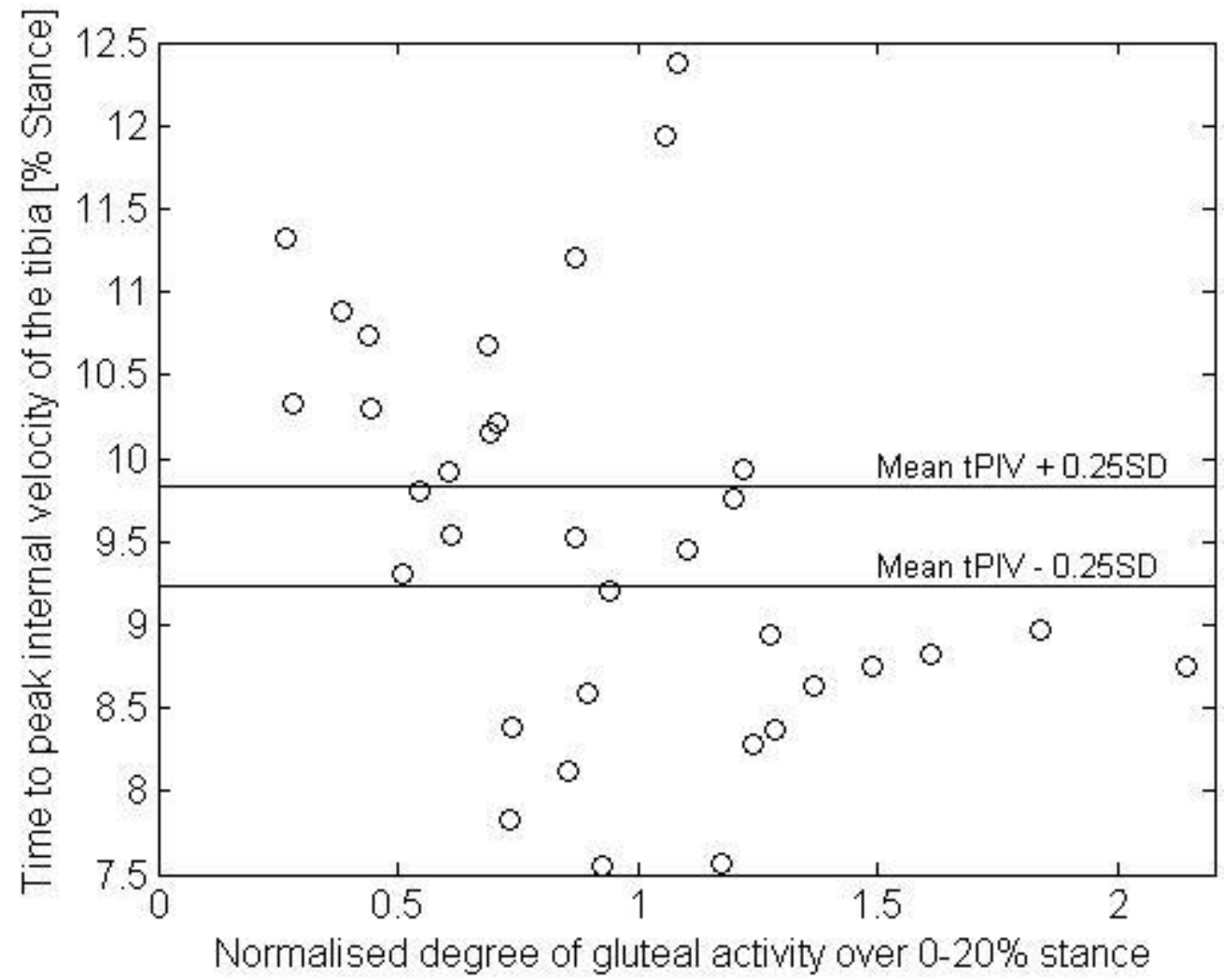

